\title{
Prevalence of diabetes and other health related problems across India and worldwide: An overview
}

\author{
U. Singh \\ Govt. Home Science College, Sector-10, Chandigarh-160010 (UT), INDIA \\ Email: usuttarasingh@gmail.com
}

Received: June 23, 2015; Revised received: December 27, 2015; Accepted: March 2, 2016

\begin{abstract}
Diabetes mellitus is a major source of mortality and morbidity along with an economic menace all over the world. In 2000, prevalence of diabetes worldwide was $171,000,000$ and in 2030, it will be 366,000,000, nearly one in ten people globally will have some form of diabetes by 2035 . There are some 382 million people living with the disease, but that could jump $55 \%$ by 2035 . The total number of people in India with diabetes to be around 50.8 million in 2010 , rising to 87.0 million by 2030 and it is estimated that by 2040 the numbers will increase upto 123.5 million. It has estimated that the prevalence of diabetes in rural populations is one-quarter that of urban population for India and other Indian sub-continent countries such as Bangladesh, Nepal, Bhutan, and Sri Lanka. Indian Council of Medical research (ICMR) revealed that a lower proportion of the population is affected in states of Northern India (Chandigarh 0.12 million, Jharkhand 0.96 million) as compared to Maharashtra (9.2 million) and Tamil Nadu (4.8 million). The National Urban Survey conducted across the metropolitan cities of India reported similar trend: 11.7 per cent in Kolkata (Eastern India), 6.1 per cent in Kashmir Valley (Northern India), 11.6 per cent in New Delhi (Northern India), and 9.3 per cent in West India (Mumbai) compared with (13.5 per cent in Chennai (South India), 16.6 per cent in Hyderabad (south India), and 12.4 per cent Bangalore (South India). Strengthening of health promotion activities in different settings, preventive health screening package, better treatment facilities and effective implementation can cure these problems worldwide.
\end{abstract}

Keywords: Diabetes, Insulin, Mortality, Morbidity, Polyuria

\section{INTRODUCTION}

Diabetes is a chronic disease that occurs either when the pancreas does not produce enough insulin or when the body cannot effectively use the insulin it produces (WHO, 2009). Diabetes is fast, becoming a leading cause of morbidity, mortality and disability across the world. Diabetes mellitus is a global metabolic epidemic affecting essential biochemical activities in almost every age group (Gupta et al., 2008). According to IDF prevalence for 2010 has risen to 285 million, representing $6.4 \%$ of the world's adult population, with a prediction that by 2030 the number of people with diabetes will have risen to 438 million corresponding to $7.8 \%$ of the adult population (IDF, 2011). India has been declared as the -Diabetic capital of world. By 2030 there would be 366 million diabetics throughout the world and 79.44 million diabetics in India alone (WHO, 2007). It is estimated that by the year 2030, diabetes is likely to be the seventh leading cause of death accounting 3.3 per cent of total deaths in the world (WHO, 2008). According to IDF $70 \%$ of the current cases of diabetes occur in low- and middle income countries. With an estimated 50.8 million people living with diabetes, India has the world's largest diabetes population, followed by China with 43.2 mil- lion. The largest age group currently affected by diabetes is between 40-59 years. By 2030 this 'record' is expected to move to the 60-79 age groups with 196 million cases (IDF, 2011). Diabetes is one of the major causes of premature illness and death worldwide. Noncommunicable diseases including diabetes account for $60 \%$ of all deaths worldwide. Type 2 diabetes is responsible for $85-95 \%$ of all diabetes in high income countries and may account for an even higher percentage in low and middle income countries. Eighty per cent of type 2 diabetes is preventable by changing diet, increasing physical activity and improving the living environment. Yet, without effective prevention and control programmes, the incidence of diabetes is likely to continue rising globally (IDF, 2011).

Diabetes increases the risk of heart disease and stroke. Fifty per cent of people with diabetes die of cardiovascular disease (primarily heart disease and stroke). Diabetes with reduced blood flow, neuropathy in the feet increases the chance of foot ulcers and eventual limb amputation. Diabetic retinopathy is an important cause of blindness and occurs as a result of long-term accumulated damage to the small blood vessels in the retina (WHO, 2011). After 15 years of diabetes, approximately $2 \%$ of people become blind and about $10 \%$ develop severe visual impairment. Diabetes is among 
the leading causes of kidney failure. Ten to twenty per cent of people with diabetes die of kidney failure. Diabetic neuropathy is damage to the nerves as a result of diabetes and affects up to $50 \%$ of people with diabetes. Although many different problems can occur as a result of diabetic neuropathy, common symptoms are tingling, pain, numbness or weakness in the feet and hands. The overall risk of dying among people with diabetes is at least double the risk of their peers without diabetes (WHO, 2011). Type 2 diabetes runs in families. Tendency is due to children learning bad habits eating a poor diet, not exercising from their parents. In general, if one has type 2 diabetes, the risk of their child getting diabetes is 1 in 7 if one was diagnosed before age 50 and 1 in 13 if diagnosed after age 50 . Child's risk is greater when the parent with type 2 diabetes is the mother. If both have type 2 diabetes then child's risk is about 1 in 2 (American Diabetes Association, 2011). Higher blood sugar levels after an overnight fast are an automatic sign of diabetes. Diabetes adjusted for the subject's age, gender, body mass index, socioeconomic status and ethnicity. People with relatively higher levels of the pesticide in their blood were having high fasting blood sugar levels (Chirag et al., 2010).

Centers for Disease Control and Prevention (CDCP) found that obesity contribute to approximately $55 \%$ of cases of type 2 diabetes and decreasing consumption of saturated fats and trans fatty acids while replacing them with unsaturated fats may decrease the risk. Weight loss is associated with reduced mortality among overweight persons with diabetes. The prevalence of overweight was $85.2 \%$ and the prevalence of obesity was $54.8 \%$ (CDCP, 2004).

Hypertension, elevated cholesterol, acromegaly, cushing's syndrome, thyrotoxicosis, chronic pancreatitis, cancer, drugs, aging and high fat diets found to increase the risk of type 2 diabetes (Jack et al., 2004). A person with first-degree relatives with type 2 diabetes have a much higher risk of developing type 2 diabetes. Concordance among monozygotic twins is close to $100 \%$ and about $25 \%$ of those with the disease have a family history of diabetes.

A person with first-degree relatives with type 2 diabetes have a much higher risk of developing type 2 diabetes. Concordance among monozygotic twins is close to $100 \%$ and about $25 \%$ of those with the disease have a family history of diabetes. Genes significantly associated with developing type 2 diabetes (Lyssenko et al., 2008 and McCarthy et al., 2010). Emotional stress like depression is a risk factor for the development of type 2 diabetes mellitus. Not only depression but general emotional stress and anxiety, sleeping problems, anger and hostility are associated with an increased risk for the development of type 2 diabetes (Knol et al., 2006). Persons who had experienced significant life events during the past five years had a 1.6-fold increased risk to have type 2 diabetes compared to those who had not experienced life events. Men who reported to have experienced two or more life events tended to have an increased risk of diabetes (Mooy et al., 2000). In America 40 to $45 \%$ persons diagnosed with diabetes have some stage of diabetic retinopathy, putting them at risk of blindness. People with type 2 diabetes have 2 to 4 times higher risk of suffering a stroke and twice to have a heart attack, it accounts for up to $50 \%$ of all diabetes deaths (WHO, 2009). The prevalence of diabetes has dramatically increased in the latter half of the $20^{\text {th }}$ century, largely due to ready availability of large quantities of calorie rich foods and the technology driven reduction in routine daily exercise (Birnbaum, 2005). Obesity and physical inactivity independently contribute to the development of type 2 diabetes. However, magnitude of risk contributed by obesity is much greater than that imparted by lack of physical activity (Rana et al., 2007).

Prevalence of diabetes in India and worldwide: Diabetes is a major threat to public health that is rapidly getting worse in the recent years. There was a $69 \%$ increase in diabetes prevalence from 1995 to 2005; exceeding the WHO estimate of a sixty per cent global increase between 1995 and 2030 and 39\% increase between 2000 and 2030 (Diabetes Atlas, 2007). The number of diabetic patients around 37 million in the country out of the total 150 million in the world and it is growing rapidly. By 2020, that number is expected to rise to 380 million and India will have 57.5 million diabetic people by the year 2025 (Diabetes Atlas, 2007). Gikas et al., (2007) concluded that the overall crude prevalence of diabetes increased significantly, from $245(8.7 \%)$ in 2002 to $358(10.3 \%)$ in 2006 $(\mathrm{P}=0.037)$. The age-adjusted prevalence of diabetes among adults was $8.2 \%$ (men, $8.5 \%$; women, $7.8 \%$ ) in 2002 and $9.5 \%$ (men, 9.7\%; women, 9.3\%) in 2006. Lee et al., (2007) conducted a study in South-East Asia and Western Pacific regions which indicates that there are 83 million individuals with diabetes and 12 countries (including Hong Kong and Taiwan) in the AsiaPacific region representing $78 \%$ of the total population of the Asia-Pacific region. Six (Australia, China, India, Tonga, South Korea and Mongolia) of ten countries with complete data reported a prevalence of diabetes exceeding and three of which (Korea, Thailand, and Tonga) have also already exceeded the WHO projections for 2030. In the 12 countries in the Asia-Pacific region, the prevalence of diabetes ranged from $2.6 \%$ to $15.1 \%$.

Kokiwar et al., (2007) conducted a study to determine the prevalence of diabetes and abnormal glucose tolerance in a rural area of Nagpur district. Nine hundred twenty four diabetic subjects aged $\geq 30$ years were selected. Thirty four $(3.67 \%)$ subjects were observed to be diabetic, $55(5.96 \%)$ had impaired glucose tolerance and $33(3.57 \%)$ had impaired fasting glycemia (IFG). A total of $122(13.20 \%)$ were observed to have abnormal glucose tolerance (AGT). Prevalence of AGT was significantly $(\mathrm{P}<0.001)$ greater in people of Raipura village, Nagpur having sedentary physical activity 
$(33.84 \%)$ as compared to people having heavy physical activity (11.53\%). Prevalence was high in those belonging to upper socioeconomic classes $(23.68 \%)$ as compared to people belonging to lower socioeconomic classes $(8.96 \%)$, in those consuming alcohol (22.29\%) as compared to nonalcoholic (11.46\%), in those having $\mathrm{BMI} \geq 25 \mathrm{~kg} / \mathrm{m} 2(27.47 \%)$ as compared to those having BMI $<25 \mathrm{~kg} / \mathrm{m} 2(9.7 \%)$, in people having family history of diabetes $(46.93 \%)$ as compared to those with no such history $(11.31 \%)$ in Raipura village, Nagpur. Boddulaa et al., (2008) reported that the highest prevalence of type 2 diabetes mellitus in developing countries occurs in the upper socio-economic group, but this has not been well documented in Indians. The age and sex standardized prevalence of diabetes in 1112 affluent adult Indian subjects was $21.1 \%$. This is the highest prevalence of diabetes reported from India. Mohan et al., (2008) presented eight year follow up data on the Chennai Urban Population Study (CUPS) cohort. Among 476 subjects who had normal glucose tolerance at the baseline $13.4 \%(n=64)$ developed diabetes and $10.1 \%(n=48)$ pre-diabetes, during the follow up period of eight years. Thirty seven individuals with impaired glucose tolerance at baseline $40.5 \%(n=15)$ developed diabetes during the same period. The overall incidence of diabetes was 20.2 per 1000 person during the follow up while the overall incidence of prediabetes was 13.1 per 1000 person during the follow up. Ramachandran et al., (2008) reported that in Tamilnadu city, diabetes increased from 13.9 to $18.6 \%$ in six years and impaired glucose tolerance decreased significantly $(\mathrm{P}<0.0001)$. The Kanchipuram town and Chennai city had similar prevalence, the periurban villages had lower diabetes prevalence, but prevalence had increased compared with that in a previous survey done in 2006. Seshiah et al., (2008) studied that a total of 4151, 3960 and 3945 pregnant women were screened in urban, semi urban and rural areas of Tamilnadu, Chennai respectively. Gestational diabetes mellitus was detected in 739 (17.8\%) women in urban, 548 $(13.8 \%)$ in semi urban and $392(9.9 \%)$ in rural areas. Out of 1679 gestational diabetes mellitus women, 1204 (72\%) were detected in first visit and the remaining $28 \%$ in subsequent visits. A significant increase $(\mathrm{P}<$ 0.0001 ) in the prevalence of gestational diabetes mellitus was observed with family history of diabetes, increased maternal age and body mass index.

Mallikarjun et al.,(2009) analyzed that the prevalence of diabetes of diabetics attending hospitals of Karnataka, India was higher among patients with diabetic mother $(25.6 \%)$ as compared to patients with diabetic father $(21.2 \%)$ and there was early onset of type -2 diabetes among patients having both parents with diabetic when compared to other patients.

According to Mehta et al., (2009) the prevalence of diabetes in urban Indian adults is about $12.1 \%$ and the prevalence of Type 2 diabetes is 4-6 times higher in urban than in rural areas. The risk factors peculiar for developing diabetes among Indians include high famil- ial aggregation, central obesity, insulin resistance and life style changes due to urbanization. Most long standing macro and micro vascular complications are also more common among Indian diabetics as compared to other races and ethnic groups. Simple interventional strategies like -eat less, eat on time and walk more can go a long way in preventing these chronic disorders among present as well as in the future generations. Mohan and Pradeepa (2009) reported an overall prevalence of diabetes in India was $2.1 \%$ in urban areas and $1.5 \%$ in rural areas in 1972 and this has rapidly climbed to $12-16 \%$ from last two decades. Today, the prevalence of diabetes in the urban metros of India is approaching the figures reported in the affluent migrant Indians. Although in rural India the prevalence of diabetes is much lower than in the urban population. Environmental and lifestyle changes resulting from industrialization and migration to urban environment from rural settings may be responsible to a large extent, for this epidemic of type 2 diabetes in Indians. The morbidity associated with type 2 diabetes would still be very high. According to Rangadhar et al., (2009) the prevalence of diabetes in rural Bengal is in between $3.5 \%$ - $5.7 \%$. Religion wise prevalence in rural Bengal shows Muslims have lowest prevalence (4.8\%) and it is highest in Hindus (5.4\%), while Christians have $(5.1 \%)$ in between them. It has been observed that food intake of peoples reflects diabetes i.e. more in meat eaters $(7.2 \%)$, most in pork eaters $(7.6 \%)$, intermediate in chicken eaters $(6.4 \%)$ and lowest in those who take goat/sheep $(6.1 \%)$. In vegetarians it is $5.8 \%$ and in fish eaters it is $5.2 \%$.

Singh (2009) conducted a study in north India to compare the prevalence of type 2 diabetes mellitus (DM) and coronary artery disease (CAD) and hypertension in DM in the rural and urban populations. Two populations (rural and urban) of the same ethnic background were randomly selected for this cross sectional survey. There were 1769 rural (894 men, 875 women) and 1806 urban subjects (904 men, 902 women) between 25-64 years of age. The survey methods included fasting and $2 \mathrm{~h}$ blood glucose and electro-cardiogram and blood pressure measurement of all subjects. Using the criteria of WHO, the prevalence of diabetes mellitus (6.0 vs $2.8 \%$ ) hypertension $(24.0$ vs $17.0 \%)$ and CAD $(9.0$ vs $3.2 \%)$ was significantly $(\mathrm{P}<0.001)$ higher in urban compared to rural subjects. Hypertension and $\mathrm{CAD}$ were significantly $(\mathrm{P}<0.001)$ more frequent among subjects with diabetes compared to non diabetes. The association of CAD and hypertension with diabetes was greater in urban than rural subjects. Excess body weight, obesity, central obesity, sedentary lifestyle, higher visible fat intake (>25 g/day); and social class 1-3 (higher and middle) were significantly $(\mathrm{P}<0.001)$ associated with diabetes. Multivariate logistic regression analysis showed that after adjustment of age, sex, body mass index, central obesity, sedentary lifestyle, higher visible fat intake and alcohol intake in men were significant risk factors of diabetes among all 
the sub-groups. According to Vijayakumar et al., (2009) a cross-sectional survey was conducted among 1990 adults (women: 1149; men: 841) in Kerala, India. Those who were already on drugs for DM and/or having fasting blood glucose (FBS) $\geq 126 \mathrm{mg} / \mathrm{dl}$ were considered as DMs; those with FBS $100-125 \mathrm{mg} / \mathrm{dl}$ were considered as impaired fasting glycaemia (IFGs). The respondents response rate was 82.7 percent during study. The crude and age adjusted prevalence of diabetes mellitus was 14.6 per cent and 12.5 per cent respectively, and that of impaired fasting glycaemia was 5.1 per cent and 4.6 per cent respectively. The crude prevalence of hypertension $(\mathrm{BP} \geq 140 / 90)$, hypercholesterolemia (fasting total serum cholesterol $\geq 200 \mathrm{mg}$ / $\mathrm{dl}$ ), central obesity (WHR $\geq 0.80$ [women] and $\geq 0.90$ [men]) was 36.1 per cent, 37.0 per cent and 85.6 per cent respectively. Gale (2010) reported that China has overtaken India as the global epicenter of the diabetes epidemic afflicts 92.4 million adults. Gale (2010) reported that China has overtaken India as the global epicenter of the diabetes epidemic afflicts 92.4 million adults. China will lose $\$ 558$ billion of national income to diabetes and heart disease between 2005 and 2015 . There are about 26.8 million diabetics in the U.S., or about 12.3 percent, 7.1 percent in India and 4.5 percent in China of the population. According to Kalra et al., (2010) the overall prevalence of type 1 diabetes in Karnal district is $10.20 / 100,000$ population, with a higher prevalence in urban $(26.6 / 100,000)$ as compared to rural areas $(4.27 / 100,000)$. Karnal has a high prevalence of type 1 diabetes $(31.9 / 100,000)$. The prevalence in men is higher $(11.56 / 100,000)$ than in women $(8.6 / 100,000)$. In the 5-16 years age group, the prevalence is $22.22 / 100,000$, while in the $0-5$ years age group, prevalence is $3.82 / 100,000$. Kumar et al., (2010) reported that diabetes is a major cause of morbidity and mortality worldwide. Prevalence of diabetes is on rise in India and it may reflect changes in lifestyle, different factors leading to the increase in diabetic prevalence and particularly localizes southern parts of India. Major complications of diabetes are neuropathy, nephropathy, coronary artery disease and retinopathy. Improper diets, immigration effects, modification in life style, inter-population differences and obesity are the principal reasons for the augmentation of the diabetic prevalence. Diabetic prevalence in Chennai, Andhra and Kerala are 15.5\%, 13.2\% and $12.4 \%$ respectively. It has been estimated that India may have 80 million diabetic people in the year of 2030.

Mahadevan et al., (2010) conducted a cross sectional study in 358 subjects aged $20-76$ years randomly selected from Chennai Urban Rural Epidemiology Study in South India. Overall prevalence of snoring and daytime sleepiness was $40 \%$ and $59 \%$, respectively. Snorers were more male, older, smokers, and had higher levels of cardio metabolic risk factors. Subjects with daytime sleepiness had higher body mass index (BMI) and abdominal obesity. Both snoring and daytime sleepiness were more prevalent among subjects with impaired glucose metabolism compared to those with normal glucose metabolism. Both sleep measures were associated with higher diabetes risk scores, as assessed by the Indian Diabetic Risk Score (IDRS). The prevalence of snoring and daytime sleepiness is high among urban south Indians and these two sleep measures are associated with glucose intolerance, metabolic syndrome, and higher diabetes risk scores. Mithal (2010) reported that twenty one per cent people of Delhi suffer from both diabetes and hypertension. Of the diabetic population in New Delhi sixty two per cent had uncontrolled diabetes with their HbA1c levels more than seven per cent. Twenty seven per cent of the diabetic patients had kidney complications. Sixty five per cent of the diabetic patients were also hypertensive and dyslipidemia, while fifty five per cent was equally high amongst both the diabetic and hypertensive populations. Indian Heart Association (2015) reported that public health estimates indicate that India will account for approximately $60 \%$ of the world's heart disease burden. According to Shaw et al., (2010) the world prevalence of diabetes among adults (aged 20-79 years) will be $6.4 \%$, affecting 285 million adults, in 2010, and will increase to $7.7 \%$ and 439 million adults by 2030 . Between 2010 and 2030, there will be a $69 \%$ increase in numbers of adults with diabetes in developing countries and a $20 \%$ increase in developed countries. Sudheer et al., (2010) reported that patients with known diabetes were carried out in Tirupati, Andhra Pradesh. Two hundred twenty two diabetic patients were inquired for a detailed questionnaire regarding the type of diabetes, life style including diet, habits, heredity and exercise. Out of them, 35 (16\%) were type I diabetic and $185(84 \%)$ were type II diabetic patients. Out of the type II diabetic patients $130(70 \%)$ fall in the age between $30-45$ years and $32(17.5 \%)$ between age $46-55$ yrs and $23(12.5 \%)$ are above 55 years. Regarding life style and habit, $45(24.5 \%)$ of the type II patients have regular habit of both smoking and alcoholic, where $15(8 \%)$ of the type II were either smokers or alcoholics alone. Among the total diabetic patients inquired, $92 \%$ of them were unaware about the role of other factors like smoking, alcohol, diet and exercise associated with diabetes.

A study on 'Healthcare Planning in North-East India; survey on Diabetes Awareness, Risk Factors and Health Attitudes in a Rural Community taken up in 2007 and published in 2009, revealed a 9\% prevalence of diabetes in persons above the age of 30 among the survey population comprising 144 persons ( 78 women and 66 men), and 44 per cent of subjects expressing concern about developing diabetes in the future, the general knowledge about diabetes was limited. Only 57 per cent of the participants knew that diabetes was not an infectious condition, and 61 per cent of the subjects did not appreciate the relationship between overweight and diabetes. Thirty one per cent of the subjects had a BMI of less than 23. The prevalence of diabetes 
in rural Tamil Nadu is increasing, and may involve 10 per cent of the population above the age of 35 within 10 years (The Hindu 2010).

\section{Conclusion}

Diabetes is an alarming disorder of the world. The prevalence of diabetes is likely to increase by the year 2025 according to the WHO projections. Currently, India is the diabetic capital of the world. In 2000, India (31.7 million) topped the world with the highest number of people with diabetes mellitus followed by China (20.8 million) with the United States (17.7 million) in second and third place respectively. Systematic programmes of walking, physical exercise and yogic practices increase insulin sensitivity and produce positive changes on physical and physiological and haematological variables of diabetic patients. Physical activity tends to increase the blood flow to the muscles resulting in increased uptake of glucose and oxygen. The effects of aerobic training on glycaemic control are well established. Adults with T2DM following a simple aerobic walking program reported a significant decrease in glycaemic parameters (HbA1c and FBG) as well as BMI and general well-being (Shenoy et al., 2010). Yogic practices when combined with other forms of physical activity should be done for 30min every day while for those individuals not having other forms of physical activity, It is recommended that yogic practices may be carried out for $45-60 \mathrm{~min}$ to achieve the metabolic benefits. Each programme has their separate influence on patients with diabetes. Abdominal obesity and family history of diabetes were found positively associated with diabetes. It was revealed that the prevalence of diabetes is increasing day by day and the increased BMI were an important predictor of diabetes.

\section{REFERENCES}

ADA, (2011). Diabetes basics, genetics of diabetes, American Diabetes Association (ADA). Retrieved April, 2011, http://www.diabetes.org/diabetes-basics/geneticsof-diabetes.html.

Boddulaa, R; Yadava, S; Bhatiaa, V; Genittaa, G; Pandeya, D; Kumara, A; Singha, H.K; Rameshb, V; Julka, S; Bansala, B; Srikanta, K. and Bhatia, E. (2008). High prevalence of type 2 diabetes mellitus in affluent urban Indians. Diabetes Res Clin Pract, 81(2): 4-7.

Birnbaum, M.J. (2005). Rejoinder: Genetic research into the causes of type 2 diabetes mellitus. Anthropology and Med, 12(6): 129-134.

CDCP (2004). Prevalence of overweight and obesity among adults with diagnosed diabetes-United States, Centers for Disease Control and Prevention, USA 1988-1994 and 1999-2002. MMWR. Morbidity and Mortality Weekly Report, 53(45): 1066-8.

Chirag, J.P; Bhattacharya, J. and Butte, A.J. (2010). An environment-wide association study (EWAS) on type 2 diabetes mellitus. PLoS ONE, 5(5): 575-582.

Diabetes Atlas (2007). The gloal burden of diabetes. Retrieved June, 2007 from https://www.idf.org/sites/ default/files/IDF_Diabetes_Atlas_2ndEd.pdf
Gale, J. (2010). China tops India on World Diabetes Ladder as Boom Spurs Obesity, Bloomberg, Businessweek.

Gikas, A; Sotiropoulos, A; Panagiotakos, D; Pastromas, V; Paraskevopoulou, E; Skliros, E. and Pappas, S. (2007). Rising prevalence of diabetes among Greek adults: Findings from two consecutive surveys in the same target population. Diabetes Res Clin. Pract., 79(2): 3259.

Gupta, R; Bajpai, K.G; John, S. and Saxena, A.M. (2008). An: overview of Indian novel traditional medicinal plants with antidiabetic potentials. African J Trod. Comp. Alt. Med., 5(1): 1-17.

International Diabetes Federation, (2011). IDF Diabetes Atlas, 4th edition Retrieved May, 2011, https:// w w w. idf.org/site s/default/files EN_6E_Atlas_Full_0.pdf.

Indian Heart Association (2015). Cardiac disease among South Asian: A silent epidemic. Retrieved December, 02, 2015 from http://indianheartassociation.org/whyindians-why-south-asians/overview/.

Jack, L; Boseman, L. and Vinicor, F. (2004). Aging Americans and diabetes. A public health and clinical response. Geriatrics, 59(4): 14-7.

Kalra, S; Kalra, B. and Sharma, A. (2010). Prevalence of type 1 diabetes mellitus in Karnal district, Haryana state, India. Diabetology and Metabolic Syndrome, 2: 14.

Kokiwar, P.R; Gupta, S. and Durge, P.M. (2007). Prevalence of diabetes in a rural area of central India. 27(1): 8-10.

Knol, M; Twisk, J; Beekman, A; Heine, R; Snoek, F. and Pouwer, F. (2006). Depression as a risk factor for the onset of type 2 diabetes: a meta-analysis. Diabetologia, 49: 837-845.

Kumar, B.D; Bhuvaneshwaran, S.P; Kumar, P.V; Mitra, A. and Mahadevappa, M. (2010). A brief description of diabetes in India. J Pharmacy Res, 3(8): 1719-1723.

Lee, C.M; Huxley, R.R; Lam, T.H; Martiniuk, A.L; Ueshema, H; Pan, W.H; Welborn, T. and Woodward, M. (2007). Prevalence of diabetes mellitus and population attributable fractions for coronary heart disease and stroke mortality in the WHO South-East Asia and Western Pacific regions. Asia Pac. J. Clin. Nutr., 16(1): 18792.

Lyssenko, V; Jonsson, A. and Almgren, P. (2008). Clinical risk factors, DNA variants, and the development of type 2 diabetes. The New Eng. J. Med., 359 (21): 2220-32.

Mahadevan, R.M. A; Mohan, D; Karunakaran, I. and Viswanathan, M. (2010). Prevalence of sleep abnormalities and their association with metabolic syndrome among Asian Indians: Chennai urban rural epidemiology study (CURES - 67). J. Diabetes Sci. and Tech., 4 (6): 223-229.

Mallikarjun, V.J; Sanjay, K; Sujata, M.J. and Shivaraj, G. (2009). Familial early onset of type-2 diabetes mellitus and its complications. North Am. J. Med. Sci., 1(7): 112 $-122$.

McCarthy, M.I; Feero, W.G. and Guttmacher, A.E. (2010). Genomics, type 2 diabetes, and obesity. The New Eng. J. Med., 363 (24): 2339-50.

Mehta, S.R; Kashyap, A.S. and Das, S. (2009). Diabetes mellitus in India: The modern scourge. Med. J. Armed Forces India, 65(1): 50-54.

Mithal, A. (2010). Two of every three diabetics in Delhi have uncontrolled diabetes: Sanofi study, Indraprastha Apollo Hospitals, one of the state coordinators of the 
sanofi-aventis SITE study in New Delhi. (http:// www.dancewithshadows.com/pillscribe/two-of-everythree-diabetics-in-delhi-have-uncontrolled-diabetessanofi-study/).

Mohan, V; Deepa, M; Anjana, R.M; Lanthorn, H. and Deepa, R. (2008). Incidence of diabetes and prediabetes in a selected urban south Indian population (CUPS-19). J. Assoc. Physicians India, 56: 152-7.

Mohan, V. and Pradeepa, R. (2009). Epidemiology of diabetes in different regions of India, Health administrator, 22(1-2): 1-18.

Mooy. J. M; De Vries, H; Grootenhuis, P.A; Bouter, L.M. and Heine, R.J. (2000). Major stressful life events in relation to prevalence of undetected type 2 diabetes. The Hoorn Study. Diabetes Care, 23: 197-201.

Ramachandran, A; Mary, S; Yamuna, A; Murugesan, N. and Snehlatha, C. (2008). High prevalence of diabetes and cardiovascular risk factors associated with urbanization in India. Diabetes Care, 31(5): 893-8.

Rana J; Tricia, Y.Li; Manson, J.E. and Frank, B. Hu. (2007). Adiposity compared with physical inactivity and risk of type 2 diabetes in women. Diabetes Care, 30: 53-58.

Rangadhar, P.B; Dinesh, K. and Analava, M. (2009). Some salient points in type 2 diabetes prevalence in rural Bengal. Ethno. Med., 3(2): 127-131.

Seshiah, V; Balaji, V; Balaji, M.S; Paneerselvam, A; Arthi, T; Thamizharasi, M. and Datta, M. (2008). Prevalence of gestational diabetes mellitus in South India (Tamil Nadu) - A community based study. J. Assoc. Physicians India, 56: 329-33.

Singh, R. (2009). Prevalence of type 2 diabetes mellitus and risk of hypertension and coronary artery disease in rural and urban population with low rates of obesity. Int. J.
Card, 66(1): 65-72.

Shaw, J. E; Sicree, R.A. and Zimmet, P.Z. (2010). Global estimates of the prevalence of diabetes for 2010 and 2030. Diabetes Res. Clin. Pract., 87(1): 4-14.

Shenoy, S; Guglani, R. and Sandhu, J.S. (2010). Effectiveness of an aerobic walking program using heart rate monitor and pedometer on the parameters of diabetes control in Asian Indians with type 2 diabetes. Prim. Care Diabetes, 4(1):41-5.

Sudheer, B; Venkatesh, S; Thilagavathi, J; Kumar, C.K.A. and Naidu, Y.V. (2010). Prevalence of diabetes in Tirupati urban population and the role of risk factors associated with it-A preliminary survey. Int. J. Pharm. Tech. Res., 2(2): 1437-1438.

The Hindu, (2010). Nine per cent prevalence of diabetes in persons above 30 among survey population. TamilNadu Vellore, Retrieved from www.hindu.com/2009/10/17/ stories/2009101752360300.html.

Vijayakumar, G; Arun, R. and Kutty, V.R. (2009). High prevalence of type 2 diabetes mellitus and other metabolic disorders in rural central Kerala. J. Assoc. Physicians India, 57: 563-67.

World Health Organization, (2007). Diabetes programme. Retrieved from http://www.who.int/diabetes/facts/world figure/en/.

World Health Organization, (2008). World health statistics. Retrieved from http://www.who.int/whosis / whostat $/ 2008$ /en/.

World Health Organization, (2009). Fact sheet no. 312: What is Diabetes? Retrieved from http://www.who.int/ mediacentre/ factsheets/fs312/en/-.

World Health Organization, (2011). Diabetes. Retrieved from http://www.who.int/mediacentre/factsheets/fs312/ en/. 